\title{
Manuali di linguistica romanza
}

La nuova collana internazionale dei Manuals of Romance Linguistics (MRL) intende fornire un panorama completo dell'intera linguistica romanza, al contempo sistematico e sintetico, che tenga conto dei più recenti risultati della ricerca.

La collana $M R L$ si prefigge di aggiornare e approfondire i contenuti delle due grandi opere finora disponibili, il Lexikon der Romanistischen Linguistik (LRL) (19882005, otto volumi in dodici tomi) e la Romanische Sprachgeschichte (RSG) (2003-2008, tre volumi), con lo scopo di integrare gli ambiti e le prospettive nuove della ricerca, in particolare quei temi finora affrontati solo a latere o in modo non sistematico.

Dal momento che non sarebbe fattibile in tempi e spazi ragionevoli una completa revisione del $L R L$ e della $R S G$, la collana dei $M R L$ si presenta in una struttura modulare e flessibile: essa prevede circa 60 volumi, ciascuno dei quali, con 15-30 contributi per un massimo di 600 pagine, affronta gli aspetti principali di un determinato tema, in modo sintetico e ben strutturato. I volumi sono concepiti in modo da essere consultabili autonomamente l'uno dall'altro, ma tali da fornire nel loro insieme uno sguardo completo ed esauriente della linguistica romanza di oggi. Il fatto che la redazione di ogni volume richieda meno tempo di quello necessario per un'opera enciclopedica della mole del $L R L$, consente di poter dar conto più agilmente e rapidamente dello status attuale delle ricerche.

I volumi sono redatti in diverse lingue - francese, italiano, spagnolo, inglese e, eccezionalmente, portoghese - ma ognuno in una sola lingua, opportunamente scelta in base al tema. L'inglese consente di dare una dimensione internazionale e interdisciplinare a temi di carattere più generale che non attengono strettamente all'ambito degli studi romanzi (per es. il Manual of Language Acquisition o il Manual of Romance Languages in the Media).

La collana $M R L$ è divisa in due grandi sezioni tematiche: 1) lingue, 2) ambiti disciplinari. Nella prima sono presentate tutte le lingue romanze (comprese le lingue creole), ciascuna in un volume a sé. La collana accorda particolare attenzione alle linguae minores che non sono state trattate finora sistematicamente in un quadro d'insieme: sono previsti perciò volumi dedicati al friulano, al corso, al gallego, ma anche un Manual of Judaeo-Romance Linguistics and Philology. La seconda sezione comprende la presentazione sistematica di tutte le sotto-discipline, tradizionali e nuove, della linguistica romanza, con un volume a parte riservato a questioni metodologiche. Particolare attenzione viene accordata alle correnti nuove e dinamiche e a settori che rivestono sempre più importanza nella ricerca e nell'insegnamento, ma che non sono stati considerati in modo adeguato nelle precedenti opere d'insieme, come per esempio le Grammatical Interfaces, le ricerche sul linguaggio dei giovani, la sociolinguistica urbana, la linguistica computazionale, la neurolinguistica, il linguaggio dei segni e la linguistica giudiziaria. Ogni volume offre per il proprio ambito un panorama ampio e ben strutturato sulla storia della ricerca e sui suoi attuali sviluppi. 
Come direttori della collana siamo lieti di aver potuto affidare l'edizione dei diversi volumi a colleghi di fama internazionale provenienti da tutti i paesi di lingue romanze e non solo. I curatori sono responsabili della concezione e della struttura dei volumi, così come della scelta degli autori dei contributi, e assicurano la presenza, accanto a una presentazione sistematica dello stato attuale delle teorie e conoscenze, anche di molte riflessioni critiche e innovative.

I diversi volumi, presi nel loro insieme, costituiscono un panorama generale ampio e aggiornato della nostra disciplina; essi sono destinati tanto a coloro che vogliano informarsi su un tema specifico quanto a coloro che desiderino abbracciare gli studi romanzi attuali nei loro molteplici aspetti. I volumi dei $M R L$ offrono inoltre un approccio nuovo e innovativo alla linguistica romanza, seguendone adeguatamente e in modo rappresentativo gli ultimi sviluppi.

Günter Holtus (Lohra/Göttingen) Fernando Sánchez-Miret (Salamanca) settembre 2019 